\title{
USDA to amend regulations on handling certain animal species
}

On 28 April 2006, the US Department of Agriculture Animal and Plant Health Inspection Service (USDA APHIS) proposed to amend the Animal Welfare Act regulations (9 CFR Part 2) regarding the handling of animals. The substance of the change adds a new paragraph to Sec. 2.131 "that would require the use of shift cages for moving and transporting potentially dangerous animals, such as big cats (lions, tigers, pumas, jaguars, and cheetahs); all species of bears; great apes (gorillas, chimpanzees, orangutans) and other nonhuman primates; and wild or exotic canids ${ }^{1}$." The purpose of this amendment is to reduce the risks and injuries that escaping animals may cause to persons and other animals, and ensure their safe transport between their enclosures.

Shift cages vary in design, some of which are permanent structures (such as connections between two enclosures), whereas others are temporary housing (such as transport cages). Regardless of the type, however, the new proposed rule would require that all shift cages meet the following performance standards:

- Attach to the animals' cage or holding pen;

- Enclose animals securely in their cages;

- Not allow animals to pass through any gaps between the shift cage and their primary enclosures;
- Be structurally sound and maintained in good repair; and

- Provide handlers access to the animals' cages without risking their safety.

The proposed rule would also require that all personnel involved in the movement or transport of potentially dangerous animals be trained in the proper use of the equipment. In addition, the rule would mandate that animal facilities establish written protocols for the safe transfer of animals.

1. UDSA APHIS. Proposed rule. Shift cage requirements. Federal Register 71(82), 25100-25102 (28 April 2006).

\section{USDA to amend National Veterinary Accreditation Program}

On 1 June 2006, USDA APHIS proposed changes to the National Veterinary Accreditation Program, a voluntary program that allows veterinarians to become certified to work with Federal veterinarians and State animal health officials. The amendment would replace the existing accreditation category with two new categories: Category I and Category II. The new accreditation Category I would limit accredited veterinarians to companion animals and related activities. These veterinarians would be able to complete certificates for the international movement of companion animals, diagnosis of exotic animal diseases in companion animals, and performance of veterinary tasks during animal disease emergencies. Category II accreditation would be more comprehensive, allowing a veterinarian to work with all animal species and on all accredited activities.

The amendment would also offer accreditation specializations and require supplemental training and periodic renewal of accreditation ${ }^{1}$. Veterinarians in either accreditation category would be required to renew their accreditation every 3 years and to complete supplemental training courses during each 3-year period.

The purpose of this amendment is to "support the [USDA's] animal health safeguarding initiatives, to involve accredited veterinarians in integrated surveil- lance activities, and to make the provisions governing our National Veterinary Accreditation Program more uniform and consistent ${ }^{1}$." In addition, the proposed rule would "increase the level of training and skill of accredited veterinarians in the areas of disease prevention and preparedness for animal health emergencies in the United States ${ }^{1}$."

Comments regarding this proposed rule change can be submitted online at http:// www.regulations.gov. USDA APHIS will consider all comments received by 31 July.

1. USDA APHIS. Proposed rule. National Veterinary Accreditation Program. Federal Register 71(105), 31109-31121 (1 June 2006). 